\title{
Klebsiella oxytoca
}

National Cancer Institute

\section{Source}

National Cancer Institute. Klebsiella oxytoca. NCI Thesaurus. Code C86455.

A species of facultatively anaerobic, Gram negative, rod shaped bacteria assigned to the phylum Proteobacteria. This species is nonmotile, oxidase negative and indole positive. $\mathrm{K}$. oxytoca is pathogenic, causing urinary tract infections, and is one of the major causes of neonatal bacteremia and septicemia. 